\title{
All Universes Great and Small
}

\author{
John D. Barrow ${ }^{1}$ and Hideo Kodama ${ }^{2}$ \\ ${ }^{1}$ DAMTP, Centre for Mathematical Sciences, \\ Wilberforce Rd., Cambridge CB3 0WA, UK \\ email: J.D.Barrow@damtp.cam.ac.uk \\ ${ }^{2}$ Yukawa Institute for Theoretical Physics, \\ Kyoto University, Kyoto 606-8502, Japan \\ email: kodama@yukawa.kyoto-u.ac.jp
}

October 27, 2018

\begin{abstract}
If the topology of the universe is compact we show how it significantly changes our assessment of the naturalness of the observed structure of the universe and the likelihood of its present state of high isotropy and near flatness arising from generic initial conditions. We also identify the most general cosmological models with compact space.
\end{abstract}

Interest has grown steadily in the possibility that the universe may be finite in volume but not possess positive spatial curvature [四]. Textbook expositions of cosmology largely ignore this possibility, but there are many reasons to contemplate the prospect of an open universe with a compact topology [2]. Many compact spaces of negative curvature can possess unusually small volumes and actions, and formulations of quantum cosmology require spatial finiteness in order for cosmological wave-functions to exist. Spacetimes that tunnel out of 'nothing' may be more likely to emerge with small compact spaces of negative curvature than as closed universes. It is interesting that the curvature radius of a compact open universe provides a characteristic length-scale to which any topological scale of periodicity might be simply related. For the more philosophically minded, the prospect of an infinite cosmological space may be unattractive because any event occurring 
here and now with finite probability must be occurring infinitely often elsewhere [3]. Observations of the expansion of the universe now imply that it will continue expanding forever, and only a compact spatial topology can reconcile this with a universe of finite volume. However, this is not the only effect of a compact topology upon the expansion of the universe. Topology is a global property that can exert a powerful local impact. Indeed, we shall see that it can introduce a radically new element into our attempts to understand the structure of the universe.

One of the imperatives of modern gravitation research has been to reconcile the observed properties of the universe with those that emerge from generic initial conditions. The general cosmological solution of Einstein's equations is specified by 4 arbitrary 3-dimensional functions of space in vacuum and by 8 such functions if a perfect fluid is present. Nonetheless, the universe we observe is within one part in $10^{5}$ of a special isotropic and spatially homogeneous $(\mathrm{SH})$ solution with zero curvature characterised by a single parameter. This surprisingly close proximity of our visible universe to a state that is isotropic and homogeneous, and almost spatially flat, is difficult to understand if matter has always obeyed conventional equations of state and exerted an attractive gravitational stress. Under such circumstances we know that isotropic expansion will not be the end-result of long-term expansion: isotropic universes are unstable. Moreover, any perturbation from exact spatial flatness will also grow as the expansion proceeds: to arrive at the present state of near flatness after 14 billion years of expansion requires a fantastic degree of initial fine tuning. A favourite resolution of these paradoxes is to give up the assumption that matter was always gravitationally attractive. If the density $\rho$ and pressure $p$, violate the strong energy condition, so that $\rho+3 p<0$, for some sufficiently long interval of time in the very early history of the universe, then the expansion will temporarily accelerate and can be driven arbitrarily close to an isotropic state of zero curvature. Such an interlude of accelerated expansion is called 'inflation' [4].

Remarkably, if an open universe possesses compact topology, many of these problems are transformed and the present state of the universe appears natural even in the absence of inflation. The simplest arena in which to make a complete analysis is provided universes which are anisotropic but spatially homogeneous. There are only nine such universes, first classified by Bianchi as types $I-I X$ [5], [6], and their general solutions possess 4 (or 8) constants in vacuum (or with perfect fluid) content, respectively. The behaviour of these universes has only been extensively studied when space is not 
compact, [7]. In non-compact space, a small neighbourhood of generic SH initial conditions containing the open Friedmann universe evolves asymptotically towards a family of anisotropic plane-wave spacetimes of Bianchi type $V I I_{h}$, whilst generic flat universes approach universes of Bianchi type $V I I_{0}$ with small expansion anisotropy and bounded curvature anisotropy. This scenario changes dramatically if the topology of space is compact because compact topology introduces severe restrictions on the anisotropies that can exist in universes with zero or negative curvature [8].

Anisotropic universes can be viewed as isotropic universes to which particular gravitational waves are added. The periodic boundary conditions that the waves must satisfy on compact negatively curved spaces are extremely restrictive because their flow is chaotic. As a result, no anisotropic open universes with compact spaces are possible at all when they contain Friedmann universes as particular cases. In particular, the Bianchi $V I I_{h}$ anisotropies that cause the generic instability [7] of open isotropic Friedmann universes with non-compact spaces can no longer exist. Remarkably, in the presence of any anisotropy at all, flat compact universes are more likely than open universes if they contain Friedmann universes as special cases. The situation in non-compact flat and open universe which inspired the invention of inflation is completely reversed. There is no flatness problem.

What are the most general SH universes when space has a compact topology? In general, the addition of spatial compactness to open or flat spaces introduces additional parameters (see Table 1). This is due to the appearance of the moduli degrees of freedom and to a decrease in the freedom of diffeomorphisms connecting physically equivalent solutions. For example, in the case of a simple 3-torus, we must specify the lattice in the Euclidean 3 -space so as to define the 3 -torus in a rotationally invariant way. We need 3 parameters to specify the lengths of 3 vectors generating the lattice and 3 parameters to specify the relative direction angles of these vectors. Hence, adding their time derivatives, we need 12 parameters. If we take into account the Hamiltonian constraint and the time translation freedom, the total number reduces to 10. By contrast, without compactness the total is only 1.

In Table 1 we see that when compactness is not assumed for open and flat spaces, the four most general SH universes are those of Bianchi types $V I_{h}, V I I_{h}, V I I I$ and $I X$ [9]. When all spaces are compact, no universes of type $V I_{h}$ can exist, whilst those of type $V I I_{h}$ must be isotropic and are no longer generic. Moreover, universes of type $I X$ which contain the Friedmann closed universes of positive curvature now cease to be among the most gen- 


\begin{tabular}{ccccc} 
Bianchi Type & \multicolumn{2}{c}{ Vacuum } & \multicolumn{2}{c}{ Perfect fluid } \\
Class A & Non-compact & Compact & Non-compact & Compact \\
$I$ & 1 & 10 & 2 & 11 \\
$I I$ & 2 & 6 & 5 & 9 \\
$V I_{0}$ & 3 & 4 & 7 & 8 \\
$V I I_{0}$ & 3 & 8 & 7 & 12 \\
$V I I I$ & 4 & $4+N_{m}$ & 8 & $8+N_{m}$ \\
$($ LRS $)$ & 2 & $2+N_{m}^{\prime}$ & 3 & $3+N_{m}^{\prime}$ \\
$I X$ & - & 4 & - & 8 \\
Class B & & & & \\
$I I I$ & 3 & $2+N_{m}^{\prime}$ & 7 & $3+N_{m}^{\prime}$ \\
$I V$ & 3 & - & 7 & - \\
$V$ & 1 & 0 & 5 & 1 \\
$V I_{h}$ & 4 & - & 8 & - \\
$V I I_{h}$ & 4 & 0 & 8 & 1
\end{tabular}

Table 1: Maximal degrees of freedom for spatially open and compact Bianchi vacuum models and for those with perfect fluid.

eral. One significant feature of the parameter count in the spatially compact open universes is the difference between the counts for vacuum and perfect fluid models. Among the spatially compact vacuum Bianchi models, the type $I$ model can be the most generic unlike in the usual situation with noncompact topology, where it is the least generic. In contrast, when a perfect fluid is present, the parameter count for type $V I I_{0}$ models is always larger than that for type $I$ models in any given space topology. Therefore, the most general locally homogeneous perfect-fluid spacetimes that include the flat isotropic model are the type $V I I_{0}$ models in the spatially compact case just as they are in the non-compact case. But when we come to ask what is the most general universe of all, a new and unusual situation emerges.

The Bianchi type VIII universes become the most general when space is compactified in a complicated way (see Table 1). In this case, the parameter count increases over the non-compact case by the number of moduli degrees of freedom, $N_{m}$. Since each compact space with the full type-VIII spatial anisotropy possesses a unique Seifert bundle structure over an orientable compact orbifold covered by the hyperbolic plane, $N_{m}$ is expressed in terms of the genus $g$ and the number, $k$, of conic singularities of the base orbifold, 


$$
N_{m}=2 k+6 g-6 .
$$

Hence the parameter count increases without bound as the topology becomes more and more complicated. What is interesting for this type is that if we restrict the model to the locally rotationally symmetric (LRS) case, the moduli degrees of freedom increases to

$$
N_{m}^{\prime}=N_{m}+2 g
$$

for the same topology. This increase can beat the decrease in the dynamical degrees of freedom describing the local geometry if $g>3$. This result is intriguing, because Bianchi type $I I I$ or VIII models do not contain Friedmann universes as special cases - although they can get arbitrarily close to them. This suggests that it is most probable for a universe to have approximate LRS type III or VIII symmetry on large scales if its space is compact and the observed region of the universe is sufficiently flat. This will be directly reflected in the pattern of the microwave background radiation on large angular scales.

We have found that topology has a major impact upon the possible forms that expanding universes can take. Almost all previous investigations of compact universes have assumed isotropic expansion. However, the introduction of any finite anisotropy reveals the impact of topology: anisotropic curved spaces are not easy to sustain. If their topology is required to be compact then the possibilities become fewer still [10].

Many questions remain to be answered. Does the introduction of inhomogeneities restrict the possible universes still further? How does the possibility of additional dimensions of space change the constraints that topology imposes upon deviations from perfectly isotropic expansion.? While at present it is very difficult to prove strong results about inhomogeneous spaces, we can expect an extension of the results in the $\mathrm{SH}$ type $V I I_{h}$ system because the rigidity theorem used to derive them was formulated in a very general way. The theorems of Borel [11] and Mostow [12 imply that a broad class

\footnotetext{
${ }^{1} \mathrm{~A}$ similar phenomenon happens for Bianchi type $I I I$. In this case the spacetime must be LRS if the space is compact, the parameter count is given by the same number as that for the LRS case of type VIII. Thus it becomes as generic as type VIII, although the non-compact counterpart is less generic.
} 
of compact spaces do not admit an anisotropic, locally homogeneous metric in any dimension [13]. This suggests a powerful extension of our results to more general situations and in higher dimensions.

If space is compact then its high level of isotropy and proximity to flatness becomes far less mysterious. Compact open universes are necessarily isotropic when they are homogeneous; flat universes are more probable than open or closed universes; and the most general universes that lie close to isotropy and homogeneity are expected to possess a complex topology whose signature may have left an indelible imprint in the microwave background radiation. Maybe a small world is simpler after all.

\section{References}

[1] G. Starkman, 1998, Class.Quant.Grav. 15, 2529

[2] G.F.R. Ellis, 1971, Gen. Rel. Gravitation 2, 7; D.D. Sokolov and V.F. Shvartsman, 1975, Sov. Phys. JETP 39, 196; M. Lachieze-Rey and J.P. Luminet, 1995, Phys. Reports 254,135

[3] G.F.R. Ellis and G. B. Brundrit, 1979, Quart. Jl. Roy. astr. Soc., 20, $37-41$

[4] A. Guth, 1981, Phys. Rev. D 23, 347

[5] L. Bianchi, 1897, Mem. Soc. It. della. Sc. (Dei. XL) (3), 11, 267.

[6] A.H. Taub, 1951, Ann. Math. 53, 472

[7] C.B. Collins and S.W. Hawking, 1973, Astrophys. J. 180, 317; A.D. Doroshkevich, V.N. Lukash and Novikov, I.D., 1973, Sov. Phys. JETP 37, 739; J.D. Barrow, 1982, Quart. Jl. Roy. astr. Soc., 23, 344; J.D. Barrow and D.H. Sonoda, 1986, Phys. Reports, 139, 1; J. Wainwright, M.J. Hancock, and C. Uggla, 1999, Class. Quantum Grav. 16, 2577; U.S. Nilsson, M.J. Hancock and J. Wainwright, 2000, Class. Quantum Grav. 17,3119 .

[8] J.D. Barrow and H. Kodama, Class. \& Quantum Grav., (in press) grqc /0012075; H. Kodama, 1998, Prog. Theor. Phys. 99, 173-236; T. Koike, M. Tanimoto and A. Hosoya, 1993, J. Math. Phys. 35, 4855; M. 
Tanimoto, T. Koike and A. Hosoya, 1997, J. Math. Phys. 38, 350 and 6557; A. Ashtekar and J. Samuel, 1991, Class.\& Quantum Grav. 8, 2191; H.V. Fagundes, 1985, Phys. Rev. Lett. 54, 1200

[9] G.F.R. Ellis and M.A.H. MacCallum, 1969, Comm. Math. Phys. 12, 108

[10] W.P. Thurston, 1982, Bull. Am. Math. Soc. 6, 357

[11] A. Borel, 1960, Ann. of Math. 72, Princeton UP. Borel's denseness theorem that if a compact space $M$ is topologically $\tilde{M} / \Gamma$ with a manifold $\tilde{M}$ admitting a homogeneous metric invariant under a transformation group $G$ isomorphic to a product of non-compact simple groups and its discrete subgroup $\Gamma$, the lift $\tilde{g}$ to $\tilde{M}$ of any metric $g$ on $M$ is $G$-invariant if $\tilde{g}$ is invariant under 1-dimensional Lie subgroup of $G$

[12] G.D. Mostow, 1973, Ann. Math. Stud. 78, Princeton UP. Mostow showed that if the centre of $G$ is a finite group and $G$ does not have a factor isomorphic to $S L(2, \mathbb{R})$, there exists no moduli freedom in the choice of $\Gamma$.

[13] Unfortunately, their physical implications to higher-dimensional cosmology are not certain at present because the most favourable compactifications in the string theory utilize Calabi-Yau manifolds, which generally admit the moduli freedom of the complex and the Kähler structures, to respect supersymmetry. However, since supersymmetry must be broken and harmful moduli fields should be eliminated in a final theory, there is a possibility that the rigidity theorem in higher dimensions may play a significant role in finding the final theory. 